\title{
Progressive myoclonus and epilepsy with dentatorubral degeneration: a clinicopathological study of the Ramsay Hunt syndrome
}

\author{
T. D. B I R D A N D C. M. S H A W
}

From the Departments of Medicine and Pathology, Divisions of Neurology, Medical Genetics, and Neuropathology, University of Washington School of Medicine, and the Seattle Veterans Administration Hospital, Seattle, Washington, USA

SUMMARY Ramsay Hunt's progressive myoclonus and epilepsy associated with dentatorubral degeneration is a rare disorder. We report a 19 year old woman with this clinical syndrome who also has a more mildly affected brother. Neuropathological examination of the young woman showed spinocerebellar and cerebral cortical degeneration in addition to dentatorubral involvement. The evidence suggests that this is a distinctive hereditary disorder producing neuronal degeneration at several levels in the central nervous system.

Progressive myoclonic cerebellar dyssynergia with epilepsy is an uncommon clinical syndrome with multiple causes. Harriman and Millar (1955) divide progressive familial myoclonic epilepsy into three categories. The first is a relatively uniform group associated with intracellular inclusions (Lafora bodies), and the second is associated with various cerebral lipidoses. The third category is a heterogeneous group of nonspecific degenerative diseases which include dyssynergia cerebellaris myoclonica of Ramsay Hunt. This syndrome has also been called dentatorubral degeneration based on the postmortem findings of the case reported by Hunt (1921). Subsequent reports of Ramsay Hunt's dentatorubral degeneration with pathological confirmation are scarce. We have evaluated a patient who seems to belong to this variety of progressive myoclonic epilepsy. The rarity and unusual nature of the syndrome justify documentation of the clinical and pathological details of this patient. In addition, other members of her family have possibly associated neurological findings which will also be described.

Supported in part by grants NS01561-01, GM13543-21 and HD02274-11 from the National Institutes of Health, US Public Health Service.

Address for correspondence and reprint requests: Thomas D. Bird, MD, Division of Neurology, University of Washington School of Medicine, Seattle, Washington 98195, USA.

Accepted 29 August 1977

\section{Clinical summary}

The patient had a normal birth and development, and was an average student. At age 15 years she developed an awkward gait, clumsy hand coordination, and deteriorating school work. She soon developed nocturnal seizure activity, and an EEG revealed irregular runs of $4-5 \mathrm{~Hz}$ moderate voltage activity over the occipital areas, with bilateral spike discharges in these same regions. She was placed on anticonvulsant drugs and six months later began to complain of dull frontal headache.

Neurological examination at this time showed marked horizontal nystagmus on lateral gaze to either side, dysarthria, slight dysmetria on fingerto-nose and heel-to-shin testing, poor rapid alternating movements with the left hand, ataxic gait, and a fine tremor of the head and hands. Deep tendon reflexes were normal and plantar responses were downgoing. Unusually high arched feet were noted.

Cerebrospinal fluid contained $11.2 \mathrm{~g} / 1$ protein, $3.3 \mathrm{mmol} / 1(60 \mathrm{mg} / \mathrm{dl})$ sugar, one mononuclear cell per $\mathrm{mm}^{3}$, and negative VDRL. Skull radiographs, pneumoencephalogram, and brain scan were normal. She was treated with a combination of mephobarbitone, diphenylhydantoin, and ethosuximide.

Three months later deep tendon reflexes were 
absent in both lower extremities and vibratory sensation was decreased in both feet. Visual fields and acuity were normal. An EEG demonstrated epileptiform discharges, predominantly over the left central and posterior regions. Repeat cerebrospinal fluid findings were unchanged and CSF protein electrophoresis was normal.

One year after the onset of symptoms the tremor and incoordination of her right hand had increased and she complained of visual blurring. Examination revealed that she was oriented and coherent, but her fund of general knowledge and calculations were impaired. Pupillary light reactions were normal. She now had vertical jerk nystagmus in addition to a worsening of her ataxic gait, dysmetria, and vibratory and position sensory deficits. Plantar reflexes remained downgoing. She had "jerky twitches" of her head and upper extremities that were described as both myoclonus and asterixis (sudden involuntary flexion, especially of the outstretched hands). Her EEG had deteriorated with posterior slow waves and diffuse epileptiform activity, worse on the left. Her verbal IQ was 85 , performance IQ 71 , and full scale IQ 77. The following tests were normal: serum sodium and potassium, complete blood count, blood sugar, blood urea nitrogen, total serum bilirubin, serum glutamic oxalacetic transaminase, serum ceruloplasmin, slit lamp examination of the eyes, urine amino acid screen, ECG, rectal biopsy, and urine porphyrins. There were no metachromatic granules in the urine. Ulnar, median, and peroneal motor nerve conduction velocities were normal but that of the posterior tibial nerve was slightly slow $(39 \mathrm{~m} / \mathrm{s})$.

The patient's disease showed a slow but relentless progression. Three years after the onset of sympioms she remained alert and oriented, but her mental capacity had deteriorated. She developed severe myoclonic jerking movements and dysmetria of all limbs. The myoclonic jerks included small twitches of individual muscles as well as sudden larger movements of a whole limb. They were often asymmetrical with the right side more involved. This jerking was worse with stimulation and movement, and decreased with rest and lethargy. There was no close correlation between the jerking of the limbs and the abnormal EEG activity. Plantar reflexes remained downgoing. Her visual acuity was poor $(20 / 160)$, and she could only identify moving hands. She had a right homonymous hemianopsia. Optic fundi and pupillary reflexes were normal. Optokinetic nystagmus was deficient in all directions but best with the stimulus moving toward her right. An EEG showed occipital delta activity, left more than right. Her generalised seizures were not well controlled with multiple anticonvulsants.

Four years after the onset of symptoms she was completely bedridden and hardly able to carry on a meaningful conversation. She had a sudden respiratory arrest and was admitted to hospital in coma without response to painful stimuli. She required intermittent assisted ventilation which maintained her arterial blood gases and $\mathrm{pH}$ within the normal range. Frequent myoclonic jerks of face and all extremities continued. She died six weeks after admission, aged 19 years.

\section{Pathological findings}

General necropsy findings included severe chronic bronchitis, moderately severe interstitial pneumonitis, severe acute and chronic cystitis, inspissated secretion of the tracheobronchial tree, and gingival hyperplasia. There was no evidence of a cardiomyopathy.

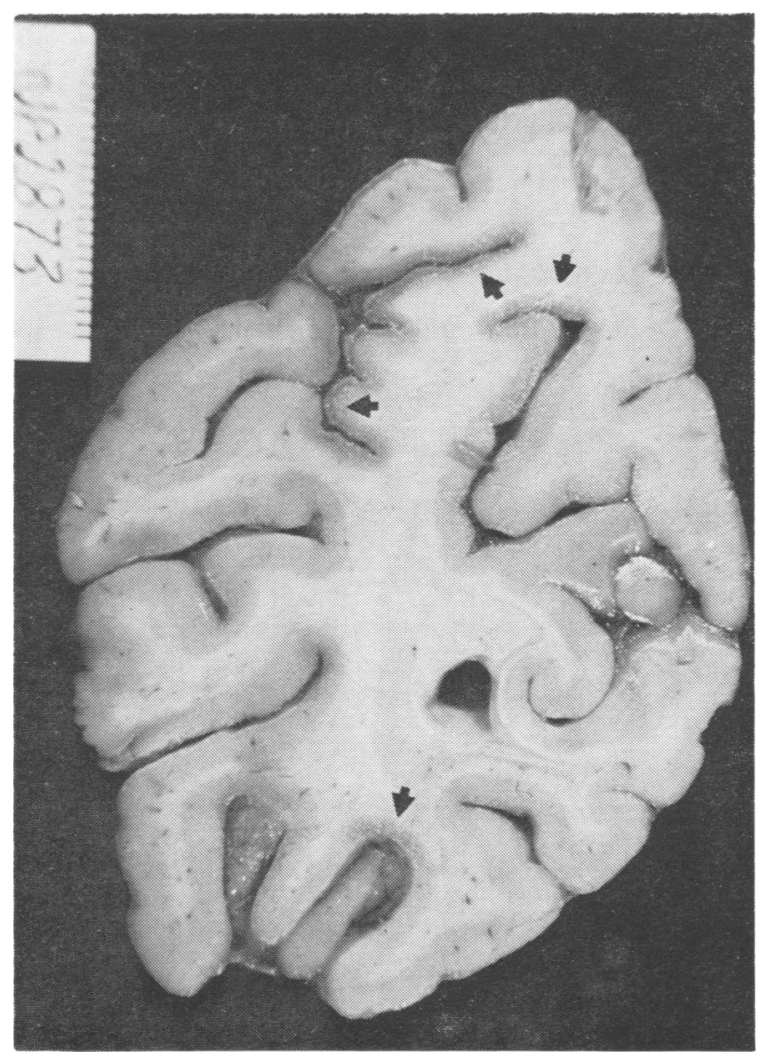

Fig. 1 Coronal section of the right parieto-occipital lobe showing cortical necrosis (arrows). 
GROSS BRAIN FINDINGS

The fresh brain weighed 1200 grams. The convolutional pattern of the cerebral hemispheres was normal but the sulci were slightly widened and the gyri were slightly narrow. The lateral ventricles were mildly enlarged. On a cut surface there were multifocal cortical lesions showing thinning of the cortex with dark brown discolouration and granularity (Fig. 1). These lesions were most marked in the medial and inferior occipital cortices, parietal cortex, inferior frontal and superior temporal gyri, more marked on the left side. The substantia nigra was pale. The cerebellum was moderately atrophic and sclerotic.

Multiple blocks from representative parts of the ccrebrum, cerebellum, and brainstem were embedded in paraffin, stained with haematoxylin and eosin, Nissl, Luxol-fast blue-periodic acid-Schiffhaematoxylin, and Holmes' axon and Holzer glial fibre methods. Samples were also taken from various areas, fixed in $4 \%$ glutaraldehyde in cacodylate buffer and processed for electron microscopic studies.

\section{MICROSCOPIC EXAMINATION}

Cerebrum Multifocal lesions of the cerebral cortex were found to be much more extensive than those noted in the gross examination, and were of various degrees of severity. In a typical lesion, the whole thickness of the cortex was re-

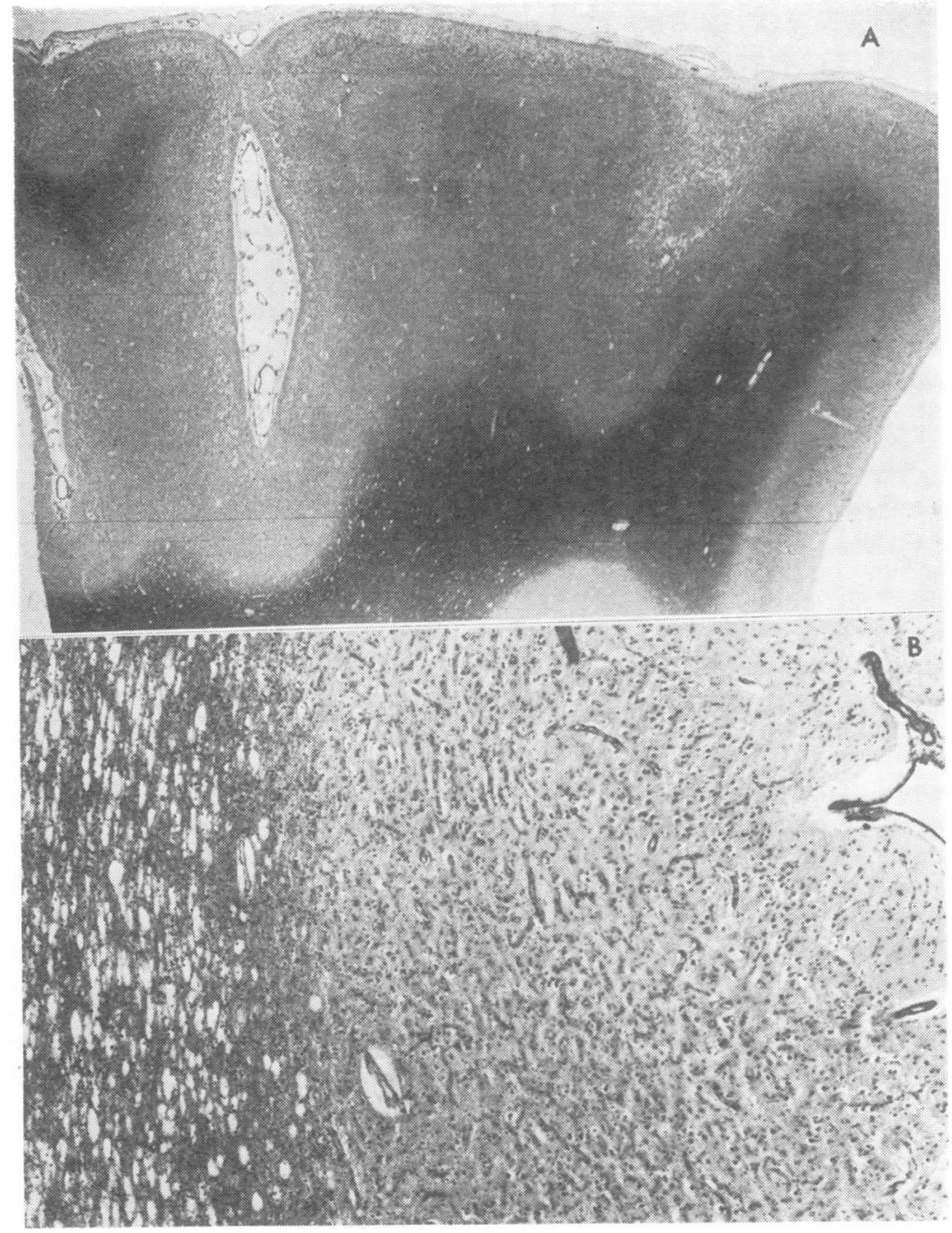

Fig. 2 Microscopic section of cerebral cortex with marked cortical necrosis $(A)$. Higher magnification $(B)$ shows cortical atrophy with neuronal loss, capillary and glial proliferation, and spongy degeneration of the subcortical white matter. LFB-PAS-H stain. 


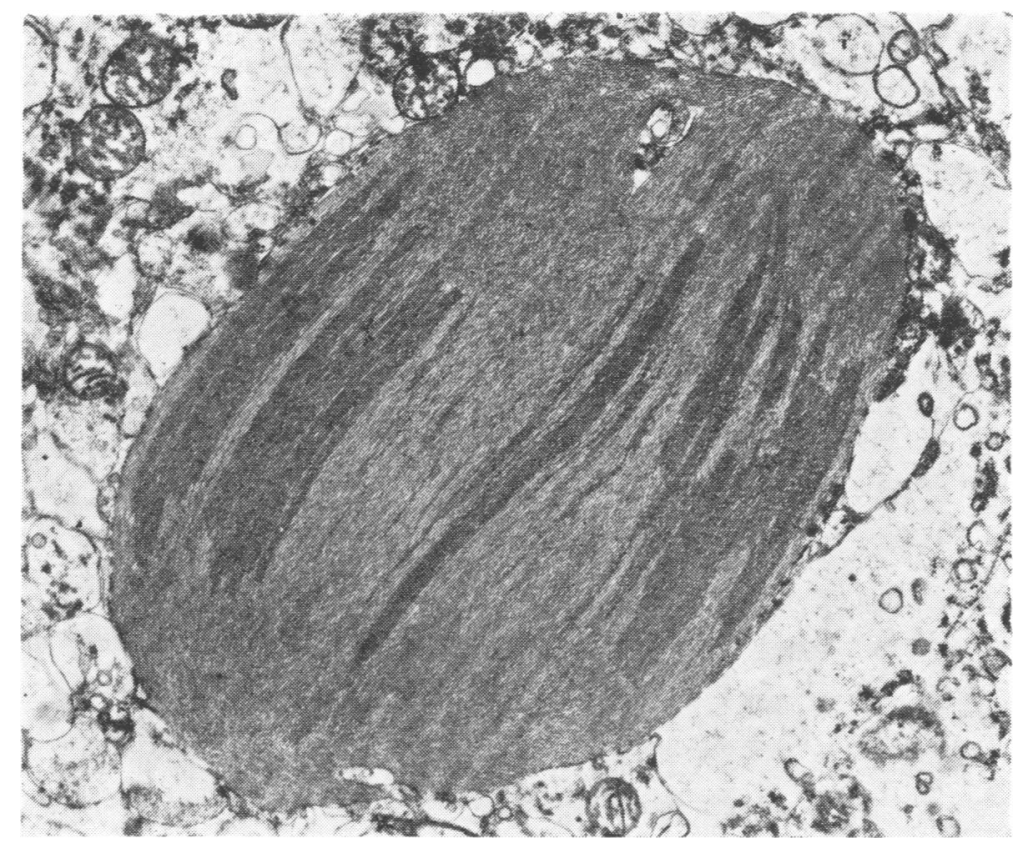

Fig. 3 Electron microscopic photographs of a rod-like body found in the hippocampus $(\times 13000)$.

placed by proliferated capillaries and hypertrophic astrocytes (Fig. 2). The deeper layers were more severely affected. Randomly scattered shrunken neurones were present in the deeper cortex but more neurones were usually preserved in layers II and III. Small vacuoles were scattered in the lesion or formed rows in layer II. Large areas of spongy degeneration were frequently present in the white matter immediately underneath the cortical lesion (Fig. 2). In the more severely affected cortex, hypertrophic astrocytes decreased in number with the relative increase of capillaries and the cortex became thin and loose with wavy ill-defined spaces. Neurones were absent. In the less severely involved cortex, focal accumulations of hypertrophic astrocytes were present with relatively focal neuronal loss. In all lesions gitter cells were infrequently found. The hippocampus and adjacent temporal cortex were spared on both sides but eosinophilic rod-like structures were found sporadically in the hippocampus. Electron microscopic studies showed these rods to be densely fibrillar structures similar to those described by Hirano et al. (1968) in Guamanian amyotrophic lateral sclerosis and Parkinsonismdementia complex (Fig. 3). However, Alzheimer's neurofibrillary change, senile plaques, and granulovacuolar change were not found in the same regions.
Basal ganglia The lenticular nuclei were normal. Focal spongy degeneration, neuronal loss and astrocytic proliferation similar to the cortical lesions were present in the dorsolateral thalamus.

Cerebellum The loss of Purkinje cells and Bergmann's gliosis were marked and widespread. The granular cells were also lost but more focally. The subcortical white matter was diffusely pale and mildly spongy. The dentate nucleus was markedly atrophic with diffuse neuronal loss and glial and capillary proliferation (Fig. 4). Only a few shrunken neurones were scattered among astrocytes and capillaries.

Brainstem There was moderate to marked degree of neuronal loss and corresponding gliosis in the substantia nigra, red nucleus, locus coeruleus, and inferior olives. Extracellular melanin pigment and its phagocytosis were evident in both substantia nigra and locus coeruleus. The brachium conjunctivum was atrophic and pale in the mesencephalon (Fig. 5A, B), and also in the rostral pontine level (Fig. 5C, D). Marked degeneration with focal accumulation of gitter cells was noted in the brachium conjunctivum at the mid pons level.

Spinal cord Marked degeneration of the dorsal 


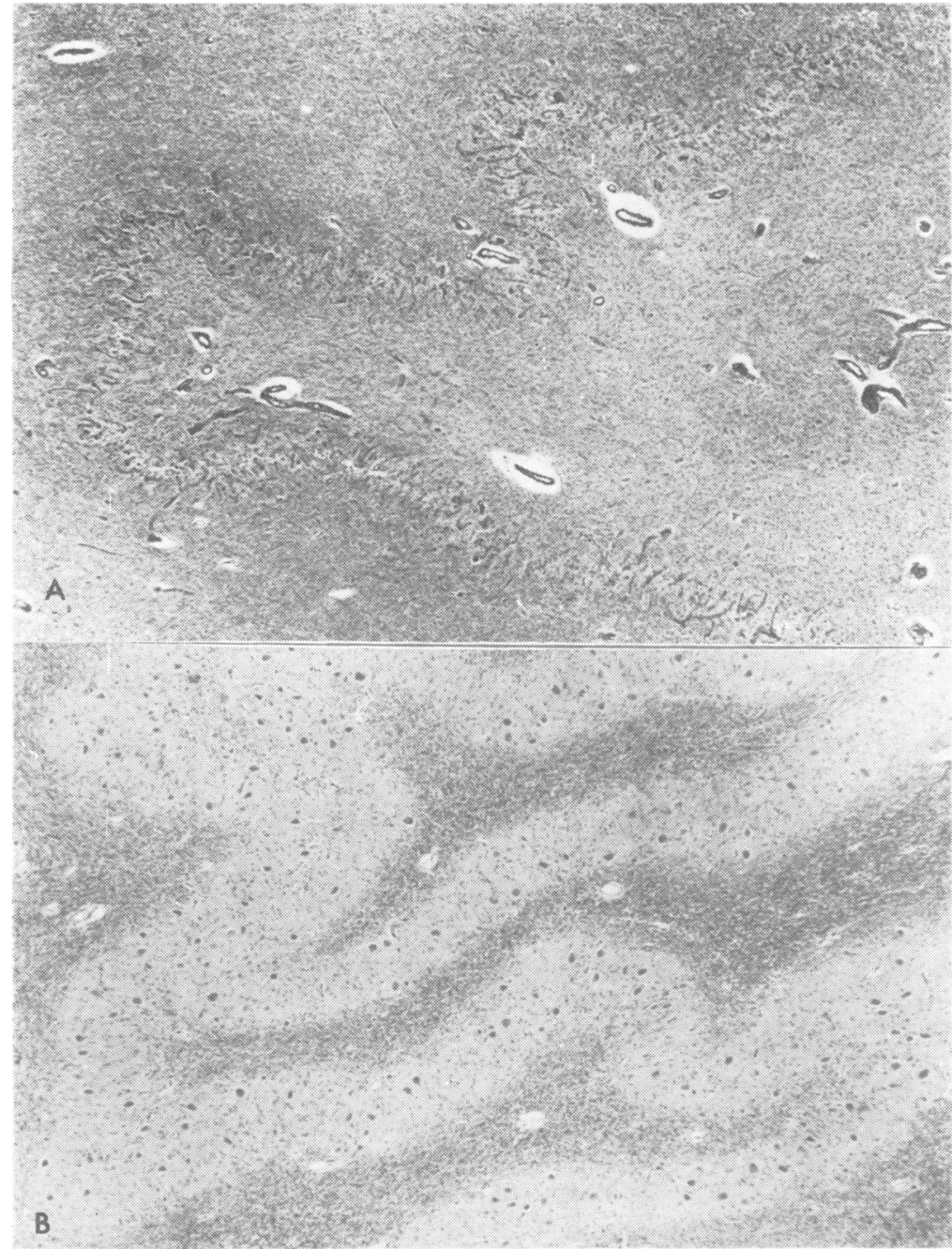

column was noted in the lumbar segments. The degeneration of the dorsal column was limited to Goll's tract in the thoracic and cervical levels where the spinocerebellar tracts were also noted to be degenerated (Fig. 6A, B). Neurones were sparse in Clarke's nucleus. The corticospinal tracts were spared. Dorsal roots in the cauda equina were diffusely fibrotic with marked loss of myelinated fibres. Only one spinal sensory ganglion from the lumbar region was available, showing cell loss and interstitial fibrosis.

No Lafora bodies were seen in the central nervous system.

\section{Family history}

The patient's father is of German ancestry, the
Fig. 4 Low power magnification of the dentate nucleus shows atrophy with marked neuronal loss, capillary proliferation, and gliosis $(A)$, compared with a normal control (B). LFB-PAS-H stain with both specimens at the same magnification.

mother of Norwegian descent, and they are unrelated. The father is living and well at age 48 years, and has had no seizures or other neurological problems. We have been unable to examine him. The 46 year old mother is healthy, has had no seizures, and has no neurological deficits on detailed examination. She has no pes cavus deformity. Her EEG shows a small amount of theta activity in the left temporal region and is otherwise unremarkable. Sensory and motor nerve conduction velocities in her upper and lower extremities are normal. She has abnormally decreased amplitudes of evoked responses $(2-3 \mathrm{mV})$ from both peroneal nerves. The patient's 23 year old brother is living and well, and has a normal neurological examination.

The patient's younger brother was normal until 


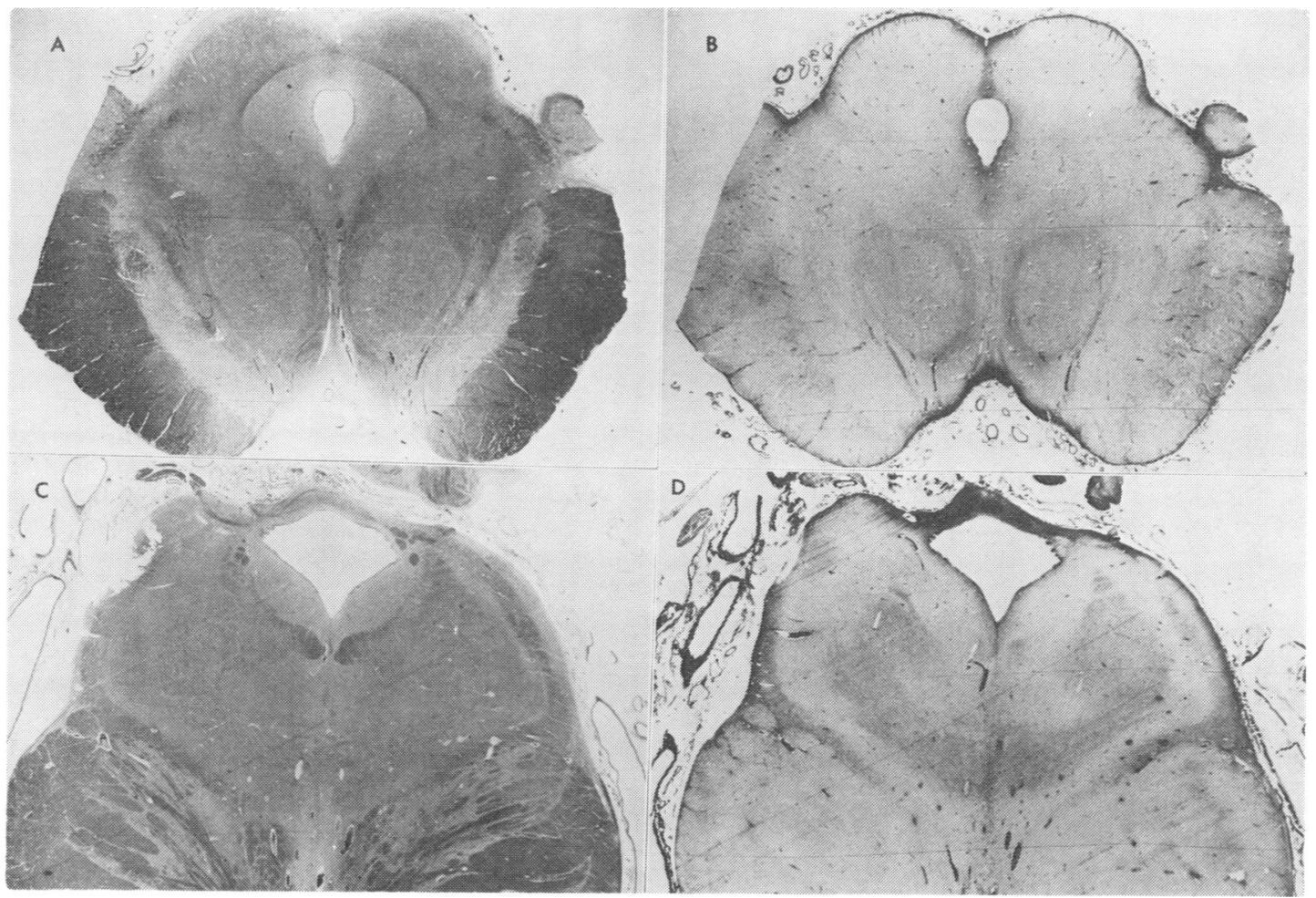

Fig. 5 Sections of the midbrain $(A, B)$ and rostral pons $(C, D)$ show marked pallor and gliosis of the brachium conjunctivum and red nucleus. Medial lemniscus and spinothalamic tracts are also involved $(C, D)$. LFB-PAS-H stain $(A, C)$ and Holzer stain $(B, D)$.

age 14 years when he developed a fine tremor of his hands and was noted to drop dishes at home. Neurological examination at the age of 17 years revealed a fine tremulousness of outstretched hands, mild pes cavus deformity, absent deep tendon reflexes, horizontal nystagmus on lateral gaze, and a loss of vibratory and position sensation in both feet. Plantar reflexes were downgoing. He has had no intellectual change, ataxia, dysarthria, seizures, or myoclonus. Electromyography showed giant motor unit potentials in the extensor digitorum brevis muscle. Ulnar and median motor nerve conduction velocities were in the borderline range but peroneal nerve conduction velocity ( 33 $\mathrm{m} / \mathrm{s})$ and distal latency $(7.9 \mathrm{~ms})$ were abnormal. Sural and median sensory nerve conduction velocities could not be obtained after considerable effort. His EEG was markedly abnormal with bilateral bursts of $3 \mathrm{~Hz}$ waves posteriorly and a moderate amount of 3-7 $\mathrm{Hz}$ arrhythmic activity over all regions bilaterally. There were sharp transients but no epileptiform activity. This EEG is almost identical to an early tracing from his sister. Urine amino acid screen was negative on this man, his mother, and his brother.

A maternal uncle of the patient has had lifelong jerking of his eyes, and was born with a strabismus that resolved spontaneously. Neurological examination at age 62 years was remarkable only for a quick oscillating horizontal movement of his eyes on forward gaze which was not made worse by eye movements. Retinae, optic discs, and maculae were normal. He had no history of seizures or myoclonus.

Another 48 year old maternal uncle had moderate nonprogressive mental retardation of unknown cause without seizures. $\mathrm{He}$ had no ataxia, tremor, sensory deficits or myoclonus. Deep tendon reflexes were normal with downgoing plantar reflexes. Rapid alternating movements were clumsy. There were a few beats of rotary nystagmus on right lateral gaze. His EEG was normal. 


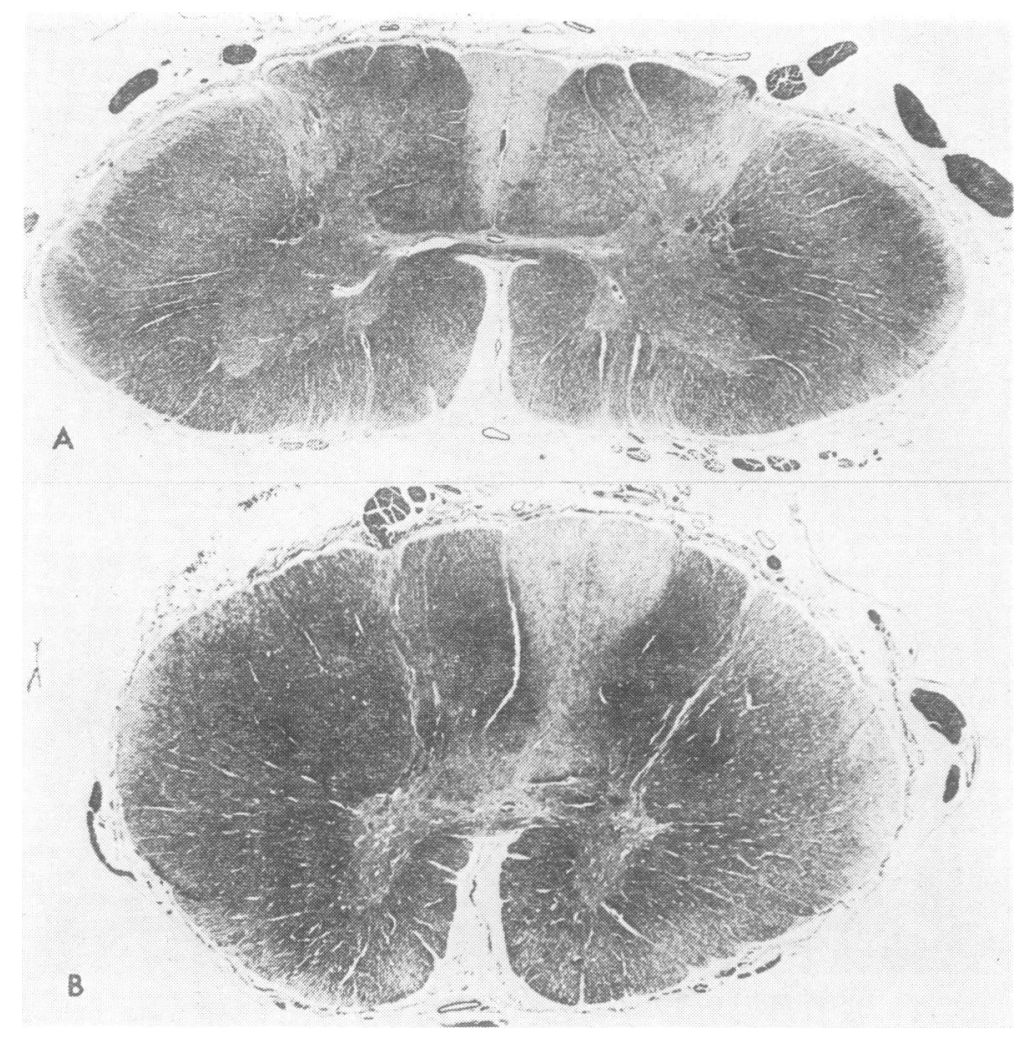

Fig. 6 Sections of the cervical cord $(A)$ and thoracic cord $(B)$ show degeneration of Goll's tract and spinocerebellar tract. LFB-PAS-H stain.

\section{Discussion}

In 1914 Ramsay Hunt reported three patients with a slowly progressive intention tremor and other evidence of cerebellar disease under the title "dyssynergia cerebellaris progressiva". No necropsy study was available at the time, but it was thought that the primary lesion was in the efferent dentate system. One of these patients came to necropsy some years later and was diagnosed as having Wilson's disease (Whittier, 1952). Critchley (1962) reported three additional cases of dyssynergia cerebellaris progressiva. The pathological findings in one of his cases included degeneration of dentatorubrothalamic pathways, subfrontal white matter, spinocerebellar, spinothalamic, spinotectal and rubrospinal tracts.

In 1921 Ramsay Hunt reported six additional patients under the title "dyssynergia cerebellaris myoclonica-primary atrophy of the dentate system". This report actually described two groups of patients. The first group consisted of four unrelated persons with progressive dysarthria, intention tremor, dysmetria, and myoclonus epilepsy.
They had no clinical evidence of spinal cord involvement and no postmortem studies. The second group consisted of one set of twin brothers (both affected) who, in addition to progressive cerebellar symptoms beginning in adolescence, also had myoclonus, epilepsy, depressed deep tendon reflexes, sensory loss in the feet, and pes cavus deformity. One of the brothers, who had signs of mental impairment, died at age 36 years. Neuropathological study showed atrophy of the dentate nucleus, degeneration of the superior cerebellar peduncle, degeneration of the posterior columns and Clarke's nucleus and the spinocerebellar tracts. The long motor (pyramidal) tracts were not involved. The cerebral cortex, red nucleus, cranial nerve nuclei, and inferior olives were normal. Ramsay Hunt described the twins as having "dyssynergia cerebellaris myoclonica associated with Friedreich's ataxia". The syndrome was later named "the Ramsay Hunt syndrome" by Greenfield (1954) but also known as dentatorubral degeneration.

Dentatorubral degeneration is a neuropathological diagnosis since the clinical syndrome of 
progressive myoclonus epilepsy is nonspecific and can be produced by numerous disorders, such as Lafora body myoclonic epilepsy, cerebral lipidoses, Creutzfeldt-Jacob disease, and even hypoxic encephalopathy. Even so, such pathological confirmations are meagre and controversial. There has not been a reported case with only dentatorubral degeneration. The few cases described include other lesions in the brainstem (Hanel and Bielschowski, and Louis-Bar and van Bogaert, both cited by Greenfield, 1954; dc Barsy et al., 1968), in the basal ganglia (Titica and van Bogaert, 1946; Andre-van Leeuwen and van Bogaert, 1949), and in the spinal cord as in the original case of Ramsay Hunt. Netsky (1968) considers these reported cases to be variants of either Friedreich's ataxia or olivopontocerebellar degeneration. In reviewing the literature on the Ramsay Hunt syndrome we also have found difficulties in defining this entity precisely.

The dentatorubral degeneration and spinal cord lesions in our patient closely resemble the findings in the single patient studied morphologically by Ramsay Hunt in 1921. Our patient differs from that of Ramsay Hunt in showing extensive cerebral cortical degeneration and degeneration in the thalamus, substantia nigra, locus coeruleus, inferior olive, and subcortical white matter. We initially interpreted the cortical lesion as being secondary to hypoxia because the patient had a respiratory arrest followed by frequent seizure activity six weeks before her death. We recognise that many observers would favour such an explanation. However, the evidence supporting the cerebral cortical lesion as a part of the primary disease is intriguing and compelling, and we favour this interpretation for the following reasons:

1. The lesion in the cerebral cortex in the present case is different from the usual hypoxic cortical necrosis or pseudolaminar necrosis in several aspects. There is practically no cystic necrosis and there are no gitter cells present in spite of evidence of a marked neuronal loss. The presence of randomly preserved neurones in the lesions is also against ischaemic necrosis. The number of hypertrophic astrocytes present in the cortex is disproportionately large. This histological lesion resembles more the degenerative process noted in other areas, such as the dentate nucleus and inferior olive. Although the cerebral cortical lesion is diffuse, it spares the inferior temporal cortex and hippocampus. This pattern is more characteristic of primary neuronal disorders, such as poliodystrophy cerebralis progressiva (Alper's disease) than of cerebral hypoxia.
2. The clinical presentation of the disease in this family includes early progressive intellectual deterioration before the respiratory arrest in the patient, and a markedly abnormal EEG in her affected brother who has not had seizures. The left sided preponderance of the cortical lesion in the patient also correlates with her EEG findings and right visual field defect.

3. Our case does not represent an isolated observation of cerebral cortical degeneration associated with familial progressive myoclonus epilepsy and dentatorubral degeneration. Similar findings have been reported by Haltia et al. (1969) and Skre and Loken (1970, case 3). The siblings reported by Morse (1949) may also represent this association.

4. Other authors have noted that focal cerebral cortical necrosis may result from persistent focal seizure activity associated with hypoxaemia that is not severe enough to produce similar damage elsewhere (Knopman et al., 1977), demonstrating that an underlying disorder of neurones may sometimes make an important contribution to the final neuropathological changes.

Based on our patient and other similar cases in the literature, we propose that dentatorubral degeneration represents the variable expression of a multifocal progressive neuronal disorder such as occurs in poliodystrophy cerebralis progressiva. The spinal cord lesions represent Wallerian degeneration from death of spinal sensory ganglia and Clarke's neurones. Degeneration of cerebral cortical neurones may or may not accompany degeneration of the dentate nucleus which was so prominent in the original case of Ramsay Hunt.

The extensive loss of cerebellar Purkinje cells could be a result of hypoxia, chronic phenytoin intoxication, part of the primary disease process, or a combination of these factors.

The Hirano bodies found in the hippocampus by electron microscopy are nonspecific changes that have been described in various degenerative diseases, including Alzheimer's disease, Parkinsonism-dementia complex, Down's syndrome, and others (Hirano et al., 1968). However, they have not been found in patients as young as this, and are unusual to be found independent of other senile changes. Degeneration of other brain stem structures, such as the red nucleus, substantia nigra, and inferior olives (as seen in our case), has been reported in other patients (Curcio and Pedace, 1955; Christophe and Gruner, 1956; Yokoi et al., 1965; de Barsy et al., 1968; Haltia et al., 1969). 
The family history of this patient is of considerable interest. Her 17 year old brother undoubtedly has the same disease, presently manifesting as absent deep tendon reflexes, pes cavus, tremulousness, mild nystagmus, decreased appreciation of vibration and position, and abnormal nerve conduction studies. His EEG is markedly abnormal and similar to his sister's. In such families the EEG may be a useful early indicator of the presence of the disease. Whether this young man will have a deteriorating clinical course remains to be seen.

No other member of this family has had a disorder similar to that of the two siblings. We conclude that this is likely to be an autosomal recessive degenerative disease, probably reflecting an inborn error of metabolism. It should be noted that both Lafora body myoclonic epilepsy and classical Friedreich's ataxia are also autosomal recessive disorders. It is possible that the mild nerve conduction abnormalities in the mother, the uncle's retardation, and the other uncle's nystagmus are mild manifestations of the recessive gene in heterozygote carriers. On the other hand, these findings could be coincidental and unrelated. A less likely possibility is that this disease is an autosomal dominant disorder with highly variable expressivity. Other reports of the Ramsay Hunt syndrome have included family pedigrees consistent with both autosomal recessive (Christophe and Gruner, 1956; Noad and Lance, 1960; Skre and Loken, 1970) and dominant inheritance (Franceschetti et al., 1954; Kreindler et al., 1959; de Barsy et al., 1968; Ziegler et al., 1974), reflecting the heterogeneity of this syndrome.

The identity of Ramsay Hunt's dyssynergia cerebellaris myoclonica remains controversial because of the scarcity of necropsied cases, the extensive overlapping of clinical manifestations with other disorders, and the nonspecific nature of myoclonus and epilepsy (Watson and Denny-Brown, 1953; Swanson et al., 1962).

The report by Ramsay Hunt (1921) of affected twin boys implies that they had two diseases: Friedreich's ataxia and dentatorubral degeneration. (It should be noted that neither the twins described by Ramsay Hunt nor our present patient had the corticospinal tract lesions found in classical Friedreich's ataxia.) We contend that those twin brothers and our present patient represent a single hereditary disorder producing primary neuronal degeneration in several levels of the nervous system. We believe Ramsay Hunt's syndrome is a specific clinicopathological disorder whose clinical features include childhood or adolescent onset, familial occurrence, progressive deterioration, myoclonic jerks, ataxia, epilepsy, abnormal electroencephalogram, hypoactive deep tendon reflexes, and diminished position and vibratory sensation. The neuropathological findings include dentatorubral and spinocerebellar degeneration associated with cortical neuronal degeneration in advanced cases. The presence of progressive intellectual deterioration and seizures, the consistently elevated CSF protein, the lack of long motor tract involvement and the absence of cardiomyopathy and scoliosis make our present patient different from typical Friedreich's ataxia (Geoff roy et al., 1976).

The Muscular Dystrophy Association aided the study of this family and Dr Robert Wilkus reviewed the electroencephalograms. Dr J. T. Robson examined one of the family members.

\section{References}

Andre-van Leeuwen, M., and van Bogaert, L. (1949). Hereditary ataxia with optic atrophy of the retrobulbar neuritis type and latent pallido-Luysian degeneration. Brain, 72, 340-363.

Christophe, J., and Gruner, J. (1956). La dyssynergie cerebelleuse myoclonique de Ramsay Hunt. Revue Neurologique. 95, 297-309.

Critchley, M. (1962). Dyssynergia cerebellaris progressiva. Transactions of the American Neurological Association, 87, 81-85.

Curcio, F. I., and Pedace, E. A. (1955). Disinergia cerebelosa mioclonica. Acta Neuropsiquiatrica Argentina. 1, 327-341.

de Barsy, T., Myle, G., Troch, C., Matthys, R., and Martin, J. J. (1968). La dyssynergie cerebelleuse myoclonique ( $R$. Hunt): affection autonome ou variante du type degeneratif de l'epilepsie-myoclonie progressive (Unverricht-Lundborg) approache anatomo-clinique. Journal of the Neurological Sciences, 8, 111-127.

Franceschetti, A., Klein, D., and Willener, H. (1954). Dyssynergie cerebelleuse myoclonique progressive (Ramsay-Hunt) a transmission dominante dans une famille bernoise. Schweitzer Archives für Neurologie und Psychiatrie, 74, 419-423.

Geoffroy, G., Barbeau, A., Breton, G., Lemieux, B., Aube, M., Leger, C., and Bouchard, J. P. (1976). Clinical description and roentgenologic evaluation of patients with Friedreich's ataxia. Le Journal Canadien des Sciences Neurologiques, 3, 279-286.

Greenfield, J. G. (1954). The Spino-cerebellar Degenerations. Charles C. Thomas: Springfield, Illinois.

Haltia, M., Kristensson, K., and Sourander, P. (1969). Neuropathological studies in three Scandinavian cases of progressive myoclonus epilepsy. Acta Neurologica Scandinavica, 45, 63-77.

Harriman, D. G. F., and Millar, J. H. D. (1955). Progressive familial myoclonic epilepsy in three 
families: its clinical features and pathological basis. Brain, 78, 325-349.

Hirano, A., Dembitzer, H. M.. Kurland, L. T., and Zimmerman, H. M. (1968). The fine structure of some intraganglionic alterations. Neurofibrillary tangles, granulovacuolar bodies and "rod-like" structures as seen in Guam amyotrophic lateral sclerosis and parkinsonian-dementia complex. Journal of Neuropathology and Experimental Neurology, 27, 167-182.

Hunt, J. R. (1914). Dyssynergia cerebellaris progressiva-a chronic progressive form of cerebellar tremor. Brain, 37, 247-268.

Hunt, J. R. (1921). Dyssynergia cerebellaris myoclonica-primary atrophy of the dentate system: a contribution to the pathology and symptomatology of the cerebellum. Brain, 44, 490-538.

Knopman, D., Margolis, G., and Reeves, A. G. (1977). Prolonged focal epilepsy and hypoxemia as a cause of focal brain damage: a case study. Annals of Neurology. 1, 195-198.

Kreindler, A., Crighel, E., and Poilici, I. (1959). Clinical and electroencephalographic investigations in myoclonic cerebellar dyssynergia. Journal of Neurology, Neurosurgery. and Psychiatry, 22, 232237.

Morse, W. I. (1949). Hereditary myoclonus epilepsytwo cases with pathological findings. Bulletin of the Johns Hopkins Hospital. 84, 116-130.

Netsky, M. G. (1968). Degenerations of the cerebellum and its pathways. In Pathology of the Ner- vous System, Volume I, pp. 1163-1185. Edited by J. Minckler. McGraw-Hill: New York.

Noad, K. B., and Lance, J. W. (1960). Familial myoclonic epilepsy and its association with cerebellar disturbance. Brain, 83, 618-630.

Skre. H., and Loken, A. C. (1970). Myoclonus epilepsy and subacute presenile clementia in heredo-ataxia. Acta Neurologica Scandinavica, 46, 18-42.

Swanson, P. D., Luttrell, C. N., and Magladery. J. W. (1962). Myoclonus-a report of 67 cases and review of the literature. Medicine (Baltimore), 41, 339-356.

Titica, J., and van Bogaert, L. (1946). Heredodegenerative hemiballismus. A contribution to the question of primary atrophy of the corpus Luysii. Brain, 69, 251-263.

Watson, C. W., and Denny-Brown, D. (1953). Myoclonus epilepsy as a symptom of diffuse neuronal disease. Archives of Neurology and Psychiatry (Chicago), 70, 151-168.

Whittier, J. R. (1952). The graphic study of ballism and related hyperkinesia. Journal of Neuropathology and Experimental Neurology, 11, 300-316.

Yokoi, S., Kobori. H., and Yoshihara, H. (1965). Clinical and neuropathological studies of myoclonic epilepsy. Acta Neuropathologica, 4, 370-379.

Ziegler, D. K., Van Speybroech, N. W.. and Seitz, E. F. (1974). Myoclonic epilepsia partialis continua and Friedreich ataxia. Archives of Neurology (Chicago), 31, 308-311. 